\title{
Optimal triangular approximation for linear stable multivariable systems
}

\author{
Diego A. Oyarzún and Mario E. Salgado
}

\begin{abstract}
This paper deals with the problem of obtaining a stable triangular approximation for a linear, square, stable, discrete-time MIMO system. We solve this problem through an analytic procedure that yields an explicit solution of a convex optimization problem. The optimized quantity is the $\mathcal{L}_{2}$ norm of the relative modelling error. An interesting feature of the proposed methodology is that, if the MIMO system has nonminimum phase zeros near the stability boundary, then the derived approximation has, at least, a set of zeros close to them. The usefulness of our result comes mainly from its use as nominal model in triangular controller design procedures based on a triangular plant model.
\end{abstract}

\section{INTRODUCTION}

In the control of some multi-input multi-output (MIMO) systems, triangular structures appear as a convenient controller structure, as happens for example in serial processes [1] and vehicle formation [2]. The design of this type of controllers is usually based on a triangular plant model [3]. One of the key reasons behind this approach is the fact that, when using a triangular nominal model, an optimal control approach for triangular controller design yields convex optimization problems [4], [5].

Choosing a triangular model for a MIMO system can be seen as a model reduction class problem, in which the term reduction refers not to lowering the order of the involved dynamical system, but to the simplification of the model structure, that is, the pattern of dependencies of plant outputs on its inputs. In the sequel, we will refer to this problem as that of triangular approximation problem.

In the current literature, the triangular approximation problem has been traditionally tackled in two stages. Firstly, by means of a suitable interaction measure such as the Relative Gain Array [6] or the Participation Matrix (PM) [3], the dominant scalar (SISO) subsystems of the MIMO system are identified. Then, in a second stage the model is obtained by eliminating those SISO subsystems that do not affect the MIMO dynamics considerably. This approach is can be seen as a triangular approximation by truncation of the original system.

The ability of the triangular model to capture some key dynamical features of the system will reflect on the degree of robustness of the design. In other words, the performance of the control loop where the triangular controller will be

D. A. Oyarzún is with Hamilton Institute, National University of Ireland, Maynooth, Ireland, previously with Department of Electronic Engineering, Universidad Técnica Federico Santa María, Valparaíso, Chile diego.oyarzun@nuim.ie

M. E. Salgado is with Department of Electronic Engineering, Universidad Técnica Federico Santa María, Valparaíso, Chile msb@elo.utfsm.cl used to control the plant will be substantially determined by the fidelity of the triangular model.

On the other hand, it has been shown [7], [8], [9] that the best achievable performance in the control of linear MIMO plants, depends on certain dynamical features of the plant. In the stable case, one of the most influential of those features is the location of the nonminimum phase (NMP) transmission zeros; in particular, in the discrete-time framework, the optimal performance is severely limited by those NMP zeros which are close to the point $z=1$. From other research work it is also known that those NMP zeros close to the stability boundary may impose fundamental control limitations [10]. It is then natural to look for a triangular approximation procedure which, in some sense, preserves these characteristics. Otherwise, the nominal triangular model will mislead the design, making highly likely that the controller will perform poorly when controlling the true plant. Of course, the most critical situation is when the controller is unable to stabilize the plant.

As a motivational example, consider the discrete-time transfer matrix

$$
\boldsymbol{G}(z)=\left[\begin{array}{cc}
z-0.5 & 0.55 \\
1 & 1
\end{array}\right] \frac{1}{z^{2}},
$$

with a PM given by

$$
\boldsymbol{\Phi}=\left[\begin{array}{cc}
0.245 & 0.1 \\
0.327 & 0.327
\end{array}\right] .
$$

The small value in $\Phi_{12}$ suggests that a lower-left triangular model should perform good as a nominal model for control design. $\boldsymbol{G}(z)$ has a single NMP zero located at $z=1.05$ and, according to the previous discussion, it is known that this zero imposes serious performance limitations and hence, it is a dynamical feature that should be taken into account in the controller design process. On the other hand, the lowerleft triangular truncation of $\boldsymbol{G}(z)$, i.e. a model identical to $\boldsymbol{G}(z)$ but with a 0 in its $(1,2)$ entry, is minimum phase, having a single zero at $z=0.5$. Hence, the information regarding the harmful NMP zero is completely lost when truncating $\boldsymbol{G}(z)$.

We focus our attention on the ability of the triangular approximation procedure to capture the nature of those NMP transmission zeros which are the key limiters of the loop performance. In this framework we must distinguish two cases:

- transmission zeros with canonical directions, that is, 
those values of $z$ which make the transfer function singular due to one or more columns or rows are entrywise zero.

- transmission zeros with non canonical directions, that is, those values of $z$ which make the transfer function singular, although no row nor column is entrywise zero.

When the triangular approximation is performed through truncation, we have that only the NMP transmission zeros with canonical directions are preserved (both location and direction). Hence, the information regarding the remaining NMP transmission zeros is generally lost in the truncation procedure.

In this paper a novel approach to the triangular approximation problem for linear, time invariant, stable and discretetime MIMO systems is presented. Namely, assuming that an interaction measure suggests a triangular structure as dominant, we do not choose the triangular model by truncation, but by minimizing the $\mathcal{L}_{2}$ norm of the relative modelling error. This optimality criterion is such that, if $\boldsymbol{G}(z)$ has NMP zeros near the stability boundary, then the optimal triangular model has, at least, a set of NMP zeros close to them. The proposed methodology is the main contribution of this work but, we also stress that, to the authors' knowledge, dealing with the triangular approximation problem with a procedure distinct from the traditional truncation is a novel approach in the literature.

The paper is organized as follows: Section II presents the notation and defines the standard concepts used throughout the article. The main result of this work is detailed in Section III. Finally, Section IV and V present an illustrative example and the concluding remarks of this work.

\section{NOTATION AND GENERAL DEFINITIONS}

We denote the set of $m \times n$ real, rational and discretetime transfer matrices by $\mathcal{R}^{m \times n}$. For any $\boldsymbol{A}(z) \in \mathcal{R}^{m \times n}$, $[\boldsymbol{A}(z)]_{i j}=A_{i j}(z)$ denotes its $(i, j)^{\text {th }}$ element and $[\boldsymbol{A}(z)]_{* j}$ denotes its $j^{t h}$ column. The transfer matrix $\boldsymbol{G}(z) \in \mathcal{R}^{p \times p}$ has a zero at $z=c \in \mathbb{C}$ if and only if $\boldsymbol{G}(c)$ is singular. This kind of zeros is usually termed in the literature as transmission zeros. If $|c| \geq 1$, the zero is called nonminimum phase (NMP), otherwise, it will be referred as minimum phase (MP). Similarly, a transfer matrix matrix having at least one NMP zero is called NMP, otherwise, is termed as MP.

The spaces $\mathcal{R} \mathcal{L}_{2}, \mathcal{R} \mathcal{H}_{2}$ and $\mathcal{R} \mathcal{H}_{2}^{\perp}$ are defined as usual [8]. The prefix $\mathcal{R}$ denotes that we restrict ourselves to the real rational case. The norm in $\mathcal{R} \mathcal{L}_{2}$ is called $\mathcal{L}_{2}$ norm and is denoted as $\|\cdot\|_{2}$. Any matrix function $\boldsymbol{A}(z) \in \mathcal{R} \mathcal{L}_{2}$ admits an orthogonal decomposition of the form

$$
\boldsymbol{A}(z)=\{\boldsymbol{A}(z)\}_{2}+\{\boldsymbol{A}(z)\}_{\perp},
$$

where $\{\boldsymbol{A}(z)\}_{2} \in \mathcal{R} \mathcal{H}_{2}$ and $\{\boldsymbol{A}(z)\}_{\perp} \in \mathcal{R} \mathcal{H}_{2}^{\perp}$, i.e. $\{\boldsymbol{A}(z)\}_{2}$ is stable and strictly proper and $\{\boldsymbol{A}(z)\}_{\perp}$ is biproper or improper having only unstable poles. Since this decomposition is orthogonal, it always holds that

$$
\|\boldsymbol{A}(z)\|_{2}^{2}=\left\|\{\boldsymbol{A}(z)\}_{2}\right\|_{2}^{2}+\left\|\{\boldsymbol{A}(z)\}_{\perp}\right\|_{2}^{2} .
$$

We say that $\boldsymbol{A}(z)$ is unitary if it satisfies $\boldsymbol{A}(z) \sim \boldsymbol{A}(z)=\boldsymbol{I}$, where $\boldsymbol{A}(z)^{\sim}=\boldsymbol{A}\left(z^{-1}\right)^{T}$ and $\boldsymbol{I}$ denotes the identity matrix. If $\boldsymbol{A}(z)$ is unitary, then it holds that $\|\boldsymbol{A}(z) \boldsymbol{M}(z)\|_{2}=$ $\|\boldsymbol{M}(z)\|_{2}$ for any $\boldsymbol{M}(z) \in \mathcal{R} \mathcal{L}_{2}$. Additionally, we denote the space $\mathcal{R H}_{\infty}$ as that of all stable and proper transfer matrices.

Every stable and biproper transfer matrix $\boldsymbol{A}(z) \in$ $\mathcal{R}^{m \times n}, m \geq n$ with full column rank for $|z|=1$ admits a factorization of the form [11]

$$
\boldsymbol{A}(z)=\boldsymbol{W}_{\boldsymbol{i}}(z) \boldsymbol{W}_{\boldsymbol{o}}(z),
$$

where $\boldsymbol{W}_{\boldsymbol{i}}(z) \in \mathcal{R}^{m \times q}$ is an inner function $\left(\boldsymbol{W}_{\boldsymbol{i}}(z) \in \mathcal{R} \mathcal{H}_{\infty}\right.$ and is unitary) and $\boldsymbol{W}_{\boldsymbol{o}}(z) \in \mathcal{R}^{q \times n}$ is an outer function (stable and right invertible in $\mathcal{R} \mathcal{H}_{\infty}$ ). If $\boldsymbol{G}(z)$ has full column rank almost everywhere (a.e.), then $\boldsymbol{W}_{\boldsymbol{o}}(z)$ is also square. Such a decomposition is known as inner-outer factorization. If $\boldsymbol{A}(z) \in \mathcal{R} \mathcal{H}_{\infty}$ is square and does not have zeros on $|z|=$ 1 , then a somewhat similar factorization can be done as

$$
\boldsymbol{A}(z)=\tilde{\boldsymbol{A}}(z) \boldsymbol{E}(z)^{-1},
$$

where $\tilde{\boldsymbol{A}}(z)$ is square, stable and minimum phase (hence, invertible in $\mathcal{R} \mathcal{H}_{\infty}$ ) and $\boldsymbol{E}(z) \in \mathcal{R} \mathcal{H}_{2}^{\perp}$ is unitary satisfying $\boldsymbol{E}(z)^{-1} \in \mathcal{R H}_{\infty}$ and $\boldsymbol{E}(1)=\boldsymbol{I}$. We will refer to $\boldsymbol{E}(z)$ as the generalized right unitary interactor (GRUI) of $\boldsymbol{A}(z)$. An algorithm to build GRUI can be found in [9].

\section{TRIANGULAR APPROXIMATION OF MIMO SYSTEMS}

This section deals with the problem of finding a triangular transfer matrix $\boldsymbol{G}_{\boldsymbol{T}}(z) \in \mathcal{R} \mathcal{H}_{\infty}$ which is close to a full MIMO transfer matrix $\boldsymbol{G}(z) \in \mathcal{R H}_{\infty}$. An essential assumption in our derivations is that some interaction measure of $\boldsymbol{G}(z)$, such as the PM defined in [3], suggests that a triangular model is a sensible choice to represent the whole MIMO dynamics.

Without loss of generality, we deal only with the case of lower-left triangular models, i.e. those models belonging to the set:

$\mathcal{T}=\left\{\boldsymbol{X}(z) \in \mathcal{R}^{p \times p}: X_{i j}(z) \equiv 0 \forall 1 \leq i \leq p, i<j \leq p,\right\}$.

In the sequel the term triangular matrix will refer to those matrices belonging to $\mathcal{T}$. Additionally, for further reference we define the triangular truncation of $\boldsymbol{G}(z) \in \mathcal{R} \mathcal{H}_{\infty}$ as $\boldsymbol{G}_{\boldsymbol{T T}}(z) \in \mathcal{R H}_{\infty}$ such that

$$
\left[\boldsymbol{G}_{\boldsymbol{T T}}(z)\right]_{i j}= \begin{cases}{[\boldsymbol{G}(z)]_{i j}} & , i=1,2, \ldots, p, j \leq i \\ 0 & , i=1,2, \ldots, p, j>i\end{cases}
$$

The fact that we are seeking for $\boldsymbol{G}_{\boldsymbol{T}}(z)$ that approaches $\boldsymbol{G}(z)$ in some sense suggests to choose $\boldsymbol{G}_{\boldsymbol{T}}(z)$ in such a way that a certain optimality criterion is minimized. The optimality index must be meaningful in terms of measuring the closeness of $\boldsymbol{G}_{\boldsymbol{T}}(z)$ and $\boldsymbol{G}(z)$, so it is natural to think in finding $\boldsymbol{G}_{\boldsymbol{T}}(z)$ such that a measure of magnitude of the modelling error is kept minimal. As a first approach, we can tackle this issue by defining the additive modelling error 
[12], [13] as

$$
\boldsymbol{G}_{\boldsymbol{\epsilon}}(z)=\boldsymbol{G}(z)-\boldsymbol{G}_{\boldsymbol{T}}(z),
$$

and solve the problem

$$
\arg \min _{\boldsymbol{G}_{\boldsymbol{T}}(z) \in \mathcal{R} \mathcal{H}_{\infty} \cap \mathcal{T}}\left\|\boldsymbol{G}_{\boldsymbol{\epsilon}}(z)\right\|_{2}^{2} .
$$

The solution to this problem can be easily obtained, as next lemma shows.

Lemma 1: Let $G(z) \in \mathcal{R} \mathcal{H}_{\infty}$, then

$$
\boldsymbol{G}_{\boldsymbol{T} \boldsymbol{T}}(z)=\arg \min _{\boldsymbol{G}_{\boldsymbol{T}}(z) \in \mathcal{R} \mathcal{H}_{\infty} \cap \mathcal{T}}\left\|\boldsymbol{G}_{\boldsymbol{\epsilon}}(z)\right\|_{2}^{2} .
$$

Proof:: From standard properties of the $\mathcal{L}_{2}$ norm we have that

$$
\left\|\boldsymbol{G}_{\boldsymbol{\epsilon}}(z)\right\|_{2}^{2}=\sum_{i=1}^{p} \sum_{j=1}^{p}\left\|\left[\boldsymbol{G}_{\boldsymbol{\epsilon}}(z)\right]_{i j}\right\|_{2}^{2} .
$$

Since $G_{T}(z) \in \mathcal{T}$, last equation yields

$$
\begin{aligned}
\left\|\boldsymbol{G}_{\boldsymbol{\epsilon}}(z)\right\|_{2}^{2}= & \sum_{i=1}^{p} \sum_{j=i+1}^{p}\left\|G_{i j}(z)\right\|_{2}^{2}+ \\
& \sum_{i=1}^{p} \sum_{j=1}^{i}\left\|G_{i j}(z)-\left[\boldsymbol{G}_{\boldsymbol{T}}(z)\right]_{i j}\right\|_{2}^{2},
\end{aligned}
$$

which can be minimized over $\mathcal{R H}_{\infty}$ by setting the second term to zero, that is, according to the definition in (8) we simply choose $\boldsymbol{G}_{\boldsymbol{T}}(z)=\boldsymbol{G}_{\boldsymbol{T} \boldsymbol{T}}(z)$.

According to last lemma, the triangular approximation that minimizes the $\mathcal{L}_{2}$ norm of the additive modelling error is precisely the triangular truncation of the plant and hence, this formulation may exhibit the inconveniences previously discussed. In other words, this approach does not capture the essential features of $G(z)$ that we want to preserve in the triangular approximation.

According to the discussion given in the Introduction, we are interested in triangular models for $\boldsymbol{G}(z)$ that preserve the information regarding the NMP zeros that are close to the stability boundary. For that purpose, we find convenient to choose a measure of the magnitude of the relative modelling error [12], [13] as the target function to be optimized. We define the relative modelling error as

$$
\boldsymbol{G}_{\boldsymbol{\Delta}}(z)=\boldsymbol{G}(z)^{-1}\left(\boldsymbol{G}_{\boldsymbol{T}}(z)-\boldsymbol{G}(z)\right),
$$

and propose finding the following optimal triangular approximation of $\boldsymbol{G}(z)$ :

$$
\begin{aligned}
\boldsymbol{G}_{\boldsymbol{T}}^{\boldsymbol{o}}(z) & =\arg \min _{\boldsymbol{G}_{\boldsymbol{T}}(z) \in \mathcal{R} \mathcal{H}_{\infty} \cap \mathcal{T}}\left\|\boldsymbol{G}_{\boldsymbol{\Delta}}(z)\right\|_{2}^{2} \\
& =\arg \min _{\boldsymbol{G}_{\boldsymbol{T}}(z) \in \mathcal{R} \mathcal{H}_{\infty} \cap \mathcal{T}} \underbrace{\left\|\boldsymbol{G}(z)^{-1} \boldsymbol{G}_{\boldsymbol{T}}(z)-\boldsymbol{I}\right\|_{2}^{2}}_{J} .
\end{aligned}
$$

Since the value of $J$ grows as the zeros of $G(z)$ approach the stability boundary, it is interesting to notice that this novel approach is equivalent to include a frequency weighting func- tion $G(z)^{-1}$ in (10) such that the minimization is stressed near the zeros of interest. The definition of $J$ requires $\boldsymbol{G}(z)$ to be invertible, which is always the case, provided that $G(z)$ is non singular a.e.. This assumption poses no severe limitation to our results since it is always satisfied when $G(z)$ has non singular DC gain, which in turn is a necessary condition for being able to track step reference signals, a standard objective in many control problems.

We emphasize that solving this optimization problem leads to solutions with NMP zeros that are close to those NMP zeros of $G(z)$ located near the stability boundary. In fact, since the $\mathcal{L}_{2}$ norm computation involves evaluating the argument in the unit circle then, if $\boldsymbol{G}(z)$ has a zero at $z=c$ near $|z|=1$, then $J$ will have a large value, unless $\boldsymbol{G}_{\boldsymbol{T}}(z)$ has a NMP zero located near $z=c$. Additionally, the cost functional $J$ is an appropriate measure of quality of the approximation since it quantifies, in a quadratic sense, the closeness of the product $\boldsymbol{G}(z)^{-1} \boldsymbol{G}_{\boldsymbol{T}}(z)$ to the identity, which is conceptually equivalent to the measure defined in (10).

Next theorem gives the solution to the problem of minimizing $J$. To keep the notation simple, let us define the $\ell^{t h}$ column of $\boldsymbol{G}_{\boldsymbol{T}}(z)$ as

$$
\left[\boldsymbol{G}_{\boldsymbol{T}}(z)\right]_{* \ell}= \begin{cases}\boldsymbol{g}_{\mathbf{1}}(z) & , \ell=1 \\
{\left[\begin{array}{ll}
\mathbf{0}_{1 \times(\ell-1)} & \boldsymbol{g}_{\boldsymbol{\ell}}(z)^{T}
\end{array}\right]^{T}} & , \ell=2,3, \ldots, p\end{cases}
$$

where $\boldsymbol{g}_{\boldsymbol{\ell}}(z) \in \mathcal{R}^{(p-\ell+1) \times 1}$. Also, as in (16), in the sequel we use a superscript $o$ to denote optimality..

Theorem 1: Let $\boldsymbol{G}(z) \in \mathcal{R} \mathcal{H}_{\infty}$ be non singular a.e., without zeros in $|z|=1$ and consider the notation defined in (16) and (17). Define the matrix $\boldsymbol{M}_{\ell}(z) \in \mathcal{R}^{p \times(p-\ell+1)}$ as the resultant from deleting the first $\ell-1$ columns of $\tilde{\boldsymbol{G}}(z)^{-1}=(\boldsymbol{G}(z) \boldsymbol{E}(z))^{-1}$, with $\boldsymbol{E}(z)$ being the GRUI of $G(z)$. Let $M_{\ell}(z)=W_{i \ell}(z) W_{o \ell}(z)$ be an inner-outer factorization of $\boldsymbol{M}_{\boldsymbol{\ell}}(z)$. Then,

$$
\boldsymbol{g}_{\ell}^{\boldsymbol{o}}(z)=z \boldsymbol{W}_{\boldsymbol{o} \ell}(z)^{-1} \boldsymbol{F}_{\mathbf{2} \ell}(z),
$$

where

$$
\boldsymbol{F}_{\mathbf{2} \ell}(z)=\left\{\frac{1}{z} \boldsymbol{W}_{\boldsymbol{i} \ell}(z)^{\sim}\left[\boldsymbol{E}(z)^{-1}\right]_{* \ell}\right\}_{2} .
$$

Proof:: We can introduce the GRUI of $\boldsymbol{G}(z)$ in $J$ such that

$$
J=\left\|\tilde{\boldsymbol{G}}(z)^{-1} \overline{\boldsymbol{G}}_{\boldsymbol{t}}(z)-\boldsymbol{E}(z)^{-1}\right\|_{2}^{2},
$$

where $\tilde{\boldsymbol{G}}(z)=\boldsymbol{G}(z) \boldsymbol{E}(z)$ is stable and invertible in $\mathcal{R} \mathcal{H}_{\infty}$, and $\boldsymbol{E}(z)$ is the associated GRUI of $\boldsymbol{G}(z)$. Using elementary properties of the $\mathcal{L}_{2}$ norm and following the notation defined in (17) it follows that

$$
J=\sum_{\ell=1}^{p}\left\|\boldsymbol{M}_{\ell}(z) \boldsymbol{g}_{\ell}(z)-\left[\boldsymbol{E}(z)^{-1}\right]_{* \ell}\right\|_{2}^{2},
$$

where the matrix $M_{\ell}(z) \in \mathcal{R}^{p \times(p-\ell+1)}$ results from deleting the first $\ell-1$ columns from $\tilde{G}(z)^{-1}$. From the definition 
of $\tilde{\boldsymbol{G}}(z)$ and the nonsingularity a.e. of $\boldsymbol{G}(z)$, it holds that $\boldsymbol{M}_{\boldsymbol{\ell}}(z)$ is stable, biproper and has full column rank a.e. Hence, we can perform an inner-outer factorization of the form $M_{\ell}(z)=\boldsymbol{W}_{\boldsymbol{i} \ell}(z) \boldsymbol{W}_{\boldsymbol{o} \ell}(z)$, with $\boldsymbol{W}_{\boldsymbol{i} \ell}(z)$ stable, unitary and biproper, and $\boldsymbol{W}_{\boldsymbol{o}}(z)$ square, stable and invertible in $\mathcal{R} \mathcal{H}_{\infty}$. Now define the unitary transfer matrix

$$
\boldsymbol{\Gamma}(z)=\left[\begin{array}{c}
\boldsymbol{W}_{\boldsymbol{i} \ell}(z)^{\sim} \\
\boldsymbol{I}-\boldsymbol{W}_{\boldsymbol{i} \ell}(z) \boldsymbol{W}_{\boldsymbol{i} \ell}(z)^{\sim}
\end{array}\right]
$$

Introducing (22) in (21) and after some algebraic manipulation it follows

$$
\begin{aligned}
J= & \sum_{\ell=1}^{p}\left\|\left(\boldsymbol{I}-\boldsymbol{W}_{\boldsymbol{i} \ell}(z) \boldsymbol{W}_{\boldsymbol{i} \ell}(z)^{\sim}\right)\left[\boldsymbol{E}(z)^{-1}\right]_{* \ell}\right\|_{2}^{2}+ \\
& \underbrace{\sum_{\ell=1}^{p}\left\|\boldsymbol{W}_{\boldsymbol{o} \ell}(z) \boldsymbol{g}_{\ell}(z)-\boldsymbol{W}_{\boldsymbol{i} \ell}(z)^{\sim}\left[\boldsymbol{E}(z)^{-1}\right]_{* \ell}\right\|_{2}^{2}}_{J^{\prime}} .
\end{aligned}
$$

According to last expression, the first sum does not depend on the unknowns, hence the vectors $\boldsymbol{g}_{\boldsymbol{\ell}}(z)$ that minimize $J$, also minimize $J^{\prime}$. We next introduce a scalar unitary factor $z^{-1}$ in $J^{\prime}$ and perform an orthogonal decomposition of the second term as

$$
\frac{1}{z} \boldsymbol{W}_{\boldsymbol{i} \ell}(z)^{\sim}\left[\boldsymbol{E}(z)^{-1}\right]_{* \ell}=\boldsymbol{F}_{\mathbf{2} \ell}(z)+\boldsymbol{F}_{\perp \ell}(z),
$$

with $\boldsymbol{F}_{\mathbf{2} \boldsymbol{\ell}}(z) \in \mathcal{R} \mathcal{H}_{2}$ and $\boldsymbol{F}_{\perp \boldsymbol{\ell}}(z) \in \mathcal{R} \mathcal{H}_{2}^{\perp}$. Substituting (24) in $J^{\prime}$ yields

$$
J^{\prime}=\sum_{\ell=1}^{p}\left\|\frac{1}{z} \boldsymbol{W}_{\boldsymbol{o} \ell}(z) \boldsymbol{g}_{\ell}(z)-\boldsymbol{F}_{\mathbf{2} \ell}(z)-\boldsymbol{F}_{\perp \ell}(z)\right\|_{2}^{2} .
$$

Since $\boldsymbol{W}_{\boldsymbol{o} \boldsymbol{\ell}}(z)$ is outer and $\boldsymbol{g}_{\boldsymbol{\ell}}(z) \in \mathcal{R} \mathcal{H}_{\infty}$ it holds that $z^{-1} \boldsymbol{W}_{\boldsymbol{o} \ell}(z) \boldsymbol{g}_{\boldsymbol{\ell}}(z)-\boldsymbol{F}_{\mathbf{2} \ell}(z)$ is stable and strictly proper, hence it belongs to $\mathcal{R} \mathcal{H}_{2}$ and an orthogonal decomposition can be done in $J^{\prime}$ as

$$
J^{\prime}=\sum_{\ell=1}^{p}\left\|\boldsymbol{F}_{\perp \boldsymbol{\ell}}(z)\right\|_{2}^{2}+\sum_{\ell=1}^{p}\left\|\frac{1}{z} \boldsymbol{W}_{\boldsymbol{o} \ell}(z) \boldsymbol{g}_{\ell}(z)-\boldsymbol{F}_{\mathbf{2} \ell}(z)\right\|_{2}^{2}
$$

The optimal vectors $\boldsymbol{g}_{\boldsymbol{\ell}}^{\boldsymbol{o}}(z)$ are such that set the second term of (26) to zero and thus (18) follows. Since $\boldsymbol{W}_{\boldsymbol{o} \ell}(z)$ is invertible in $\mathcal{R H}_{\infty}$ and $\boldsymbol{F}_{\mathbf{2} \boldsymbol{\ell}}(z)$ is stable and strictly proper, then $\boldsymbol{g}_{\boldsymbol{\ell}}(z) \in \mathcal{R} \mathcal{H}_{\infty}$ and $\boldsymbol{G}_{\boldsymbol{T}}(z) \in \mathcal{R} \mathcal{H}_{\infty}$. of the optimal triangular approximation $\boldsymbol{G}_{\boldsymbol{T}}(z)$ is always identical to that of $\boldsymbol{G}(z)$. Indeed, using Theorem 1 with $\ell=1$, i.e. we are interested in determining the first column of $\boldsymbol{G}_{\boldsymbol{T}}(z)$, it follows that $\boldsymbol{M}_{\mathbf{1}}(z)=\tilde{\boldsymbol{G}}(z)^{-1}=(\boldsymbol{G}(z) \boldsymbol{E}(z))^{-1}$, which is stable and invertible in $\mathcal{R H}_{\infty}$ and hence, an outer transfer matrix. Thus, it follows that $\boldsymbol{W}_{\boldsymbol{i 1}}(z)=\boldsymbol{I}$,

$$
\begin{aligned}
& \boldsymbol{W}_{\boldsymbol{o} \mathbf{1}}(z)=\tilde{\boldsymbol{G}}(z)^{-1} \text { and } \\
& \qquad \begin{aligned}
\boldsymbol{g}_{\mathbf{1}}^{\boldsymbol{o}}(z) & =z \tilde{\boldsymbol{G}}(z)\left\{\frac{1}{z}\left[\boldsymbol{E}(z)^{-1}\right]_{* 1}\right\}_{2}, \\
& =[\boldsymbol{G}(z)]_{* 1},
\end{aligned}
\end{aligned}
$$

which confirms our initial statement.

The procedure of Theorem 1 allows to obtain a triangular reduced model of a stable MIMO system. The main advantage of this result over the traditional triangular truncation is that, if $\boldsymbol{G}(z)$ has any non canonical NMP zeros near the unit circle, then (some of) the zeros of $\boldsymbol{G}_{\boldsymbol{T}}$ will certainly be close to them. This feature, as previously discussed, may not be attained by a triangular truncation $\boldsymbol{G}_{\boldsymbol{T} \boldsymbol{T}}(z)$.

From a controller design point of view, using $\boldsymbol{G}_{\boldsymbol{T}}(z)$ as nominal model rather that $\boldsymbol{G}_{\boldsymbol{T} \boldsymbol{T}}(z)$ can have an important impact on the performance of the real control loop. The key issue behind this idea is that, since $\boldsymbol{G}_{\boldsymbol{T}}(z)$ has information on those dynamical features of $\boldsymbol{G}(z)$ that impose performance limitations, the designed control law will be, in general, more conservative than a design that uses $\boldsymbol{G}_{\boldsymbol{T} \boldsymbol{T}}(z)$ as nominal model. This is turns implies that the real loop is more likely to perform as the nominal design when using $\boldsymbol{G}_{\boldsymbol{T}}(z)$ rather than $\boldsymbol{G}_{\boldsymbol{T} \boldsymbol{T}}(z)$ as model.

An insightful way of assessing the advantages of this procedure with respect to triangular truncation, is to compare the best achievable performance for $\boldsymbol{G}(z), \boldsymbol{G}_{\boldsymbol{T}}(z)$ and $\boldsymbol{G}_{\boldsymbol{T} \boldsymbol{T}}(z)$ using the results in [9]. In the context of this benchmark, if we denote these quantities as $J_{\text {opt }}$, $J_{\text {Topt }}$ and $J_{\text {TTopt }}$, respectively, the proposed approach will hopefully lead to

$$
\left|J_{\text {opt }}-J_{\text {Topt }}\right|<\left|J_{\text {opt }}-J_{\text {TTopt }}\right| .
$$

The previous inequality is an indicator of effectiveness of this approach. Additionally, it should be pointed out that our procedure may yield a triangular model $\boldsymbol{G}_{\boldsymbol{T}}(z)$ with additional zeros and/or poles which are not present in $\boldsymbol{G}(z)$. This feature appears inherently in the minimization of the proposed cost functional and, if these extra zeros are NMP, then the design will turn to be even more conservative.

Remark 1: The results presented in this paper deal only with the case of lower-left triangular models (i.e., the $\mathcal{T}$ set). However, it is worth mentioning that there is no loss of generality in our approach since, if another triangular structure is suggested by the PM, then we can always perform a set of input/output permutations such that the permuted system has a PM suggesting a model in $\mathcal{T}$.

To illustrate the proposed triangular approximation procedure, an example is presented next.

\section{EXAMPLE}

Consider the system

$$
\boldsymbol{G}(z)=\left[\begin{array}{cc}
z-0.5 & a \\
1 & 1
\end{array}\right] \frac{1}{z^{2}}
$$

This system has a zero at $z=a+0.5$, so that if $a>0.5$, that zero is NMP. In this case, $\boldsymbol{G}_{\boldsymbol{T} T}(z)$ is MP for any value of $a$. Hence, when the NMP zero approaches $z=1$, the 
model $\boldsymbol{G}_{\boldsymbol{T} \boldsymbol{T}}(z)$ lacks key input/output information on $\boldsymbol{G}(z)$. We can apply the procedure of Theorem 1 in order to obtain a more accurate triangular model of $\boldsymbol{G}(z)$. For instance, if $a=0.55$ then the zero is located at $z=1.05$ (this is the same case as in the introduction of this paper) and the PM suggests that a lower left triangular model is sensible. Using Theorem 1 yields

$$
\boldsymbol{G}_{\boldsymbol{T}}(z)=\left[\begin{array}{cc}
\frac{(z-0.5)}{z^{2}} & 0 \\
\frac{1}{z^{2}} & \frac{-0.034(z+6.27)(z-3.436)(z-1.046)}{z^{2}(z-0.365)}
\end{array}\right]
$$

From this result we have that indeed, $\boldsymbol{G}_{\boldsymbol{T}}(z)$ has a zero near $z=1.05$, namely at $z=1.046$. Also, as mentioned before, three additional poles and two NMP zeros appear in the optimal triangular approximation. It can be verified that, if the optimal performance triangular controller ${ }^{1}$ of [?] is used to control $\boldsymbol{G}(z)$, the real loop is unstable when using $\boldsymbol{G}_{\boldsymbol{T T}}(z)$ as nominal model, but this problem is overcome when the design is based on $\boldsymbol{G}_{\boldsymbol{T}}(z)$.

The closeness of the smallest NMP zero of $\boldsymbol{G}_{\boldsymbol{T}}(z)$ to the one of $\boldsymbol{G}(z)$ is shown in Fig. 1 as a function of the zero location. It can there be noticed that this deviation increases as the zero moves away from the unit circle, which is consistent with our previous discussion on the properties of the minimized cost functional. Additionally, the best achievable performance for $\boldsymbol{G}(z)$ and $\boldsymbol{G}_{\boldsymbol{T}}(z)$ are computed using the results in [9] as a function of the zero location. The relative difference between both quantities is plotted in Fig. 2(b). Note that in this example the optimal performance for $\boldsymbol{G}_{\boldsymbol{T T}}(z)$ is given by $J_{T T o p t}=3$, independently of $a$. A simple comparison between Figs. 2(a) and 2(b) confirms the inequality (29). It is worth mentioning that it may be verified that, in all the range spanned by $a$ in Figs. 1 and 2, the PM suggests that a lower left triangular model is sensible, so that our essential assumption holds.

\section{Conclusions}

In this paper a method for deriving a triangular model of a given stable MIMO transfer matrix is proposed. The approach is novel in the literature in the sense that the triangular model of the plant is chosen such that a measure of the modelling error is kept minimal. Unlike the classical truncation method, which gives a model that usually loses important dynamical features of the plant, the proposed approximated model preserves key information regarding the nonminimum phase zeros close to the stability boundary. Since these zeros impose severe limitations to the performance of a closed loop control scheme, this feature can be significant if the model is used for control design purposes.

Further research should explore additional properties of the solution, such as the appearance of extra zeros and/or poles in the optimal solution, and the extension to the case of unstable plants. Also, it is noted that the proposed procedure,

\footnotetext{
${ }^{1}$ This optimal triangular controller is such that minimizes the $\mathcal{L}_{2}$ norm of the loop sensitivity function and provides integral action.
}

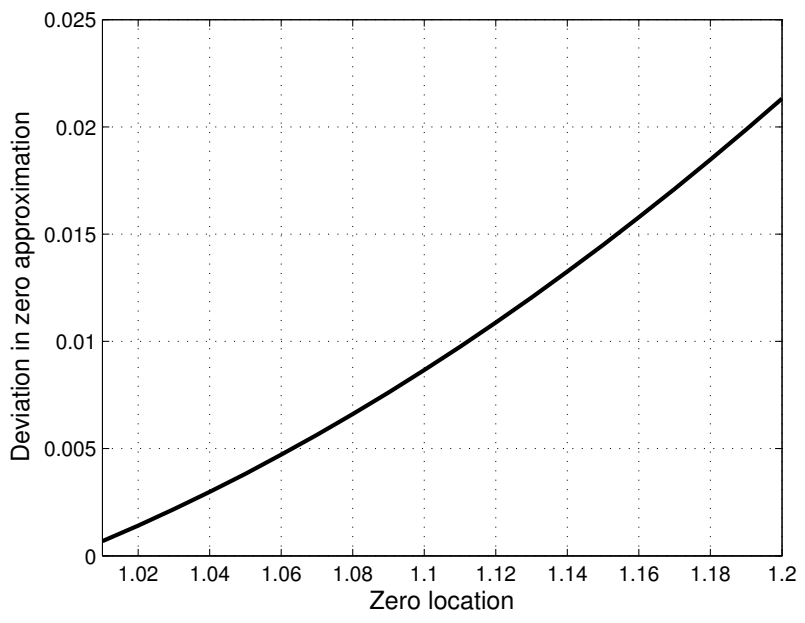

Fig. 1. Absolute deviation of the NMP zero of $\boldsymbol{G}_{\boldsymbol{T}}(z)$ with respect to $z=a+0.5$.
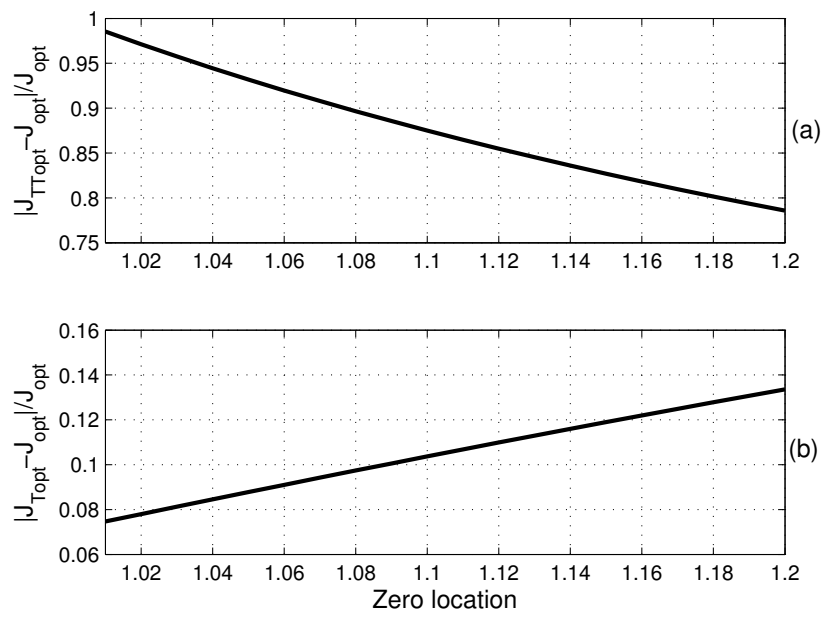

Fig. 2. Relative difference of the best achievable performance for: (a) $\boldsymbol{G}(z)$ and, (b) $\boldsymbol{G}_{\boldsymbol{T} \boldsymbol{T}}(z)$, with respect to the one of $\boldsymbol{G}(z)$.

with mild modifications, can also be used to derive sparse structured approximations other than triangular.

\section{ACKNOWLEDGEMENTS}

The authors gratefully acknowledge the support received from Universidad Técnica Federico Santa María and from CONICYT through grant Fondecyt 1060437.

\section{REFERENCES}

[1] A. Faanes and S. Skogestad, "Controller design for serial processes," Journal of Process Control, vol. 15, pp. 259-271, 2005.

[2] X. Qi, M. Salapaka, P. Voulgaris, and M. Khammash, "Structured optimal and robust control with multiple criteria: A convex solution," IEEE Transactions on Automatic Control, vol. 49, no. 10, pp. 1623 1640, October 2004.

[3] M. Salgado and A. Conley, "MIMO interaction measure and controller structure selection," International Journal of Control, vol. 77, no. 4, pp. 367-383, March 2004.

[4] M. Rotkowitz and S. Lall, "A characterization of convex problems in decentralized control," IEEE Transaction on Automatic Control, vol. 51, pp. 274-286, 2006. 
[5] E. Silva, D. Oyarzún, and M. Salgado, "On structurally constrained $\mathcal{H}_{2}$ performance bounds for stable MIMO plant models," To appear in IET Control Theory \& Applications, 2007.

[6] E. H. Bristol, "On a new measure of interaction for multivariable process control," IEEE Transactions on Automatic Control, vol. 11, pp. 133-134, 1966.

[7] J. Chen, L. Qiu, and O. Toker, "Limitations on maximal tracking accuracy," IEEE Transactions on Automatic Control, vol. 45, no. 2, pp. 326-331, 2000.

[8] O. Toker, L. Chen, and L. Qiu, "Tracking performance limitations in LTI multivariable discrete-time systems," IEEE Transactions on Circuits and Systems-Part I: Fundamental Theory and Applications, vol. 49, no. 5, pp. 657-670, May 2002.

[9] E. Silva and M. Salgado, "Performance bounds for feedback control of nonminimum-phase MIMO systems with arbitrary delay structure," IEE Proceedings - Control Theory and Applications, vol. 152, no. 2, pp. 211-219, March 2005.

[10] M. M. Seron, J. H. Braslavsky, and G. C. Goodwin, Fundamental Limitations in Filtering and Control. London: Springer Verlag, 1997.

[11] B. Francis, A Course on $H_{\infty}$ Control Theory, ser. Lecture Notes in Control and Information Sciences. New York: Springer Verlag, 1987, vol. 8.

[12] K. Zhou and J. Doyle, Essentials of Robust Control. Englewood Cliffs, New Jersey: Prentice Hall, 1998.

[13] G. C. Goodwin, S. Graebe, and M. E. Salgado, Control System Design. New Jersey: Prentice Hall, 2001. 